\title{
Selective Metal-Phenolic Assembly from Complex Multicomponent Mixtures
}

Gan Lin,${ }^{\dagger}$ Md. Arifur Rahim, ${ }^{\dagger}$ Michael G. Leeming, ${ }^{\dagger}$ Christina Cortez-Jugo, ${ }^{\dagger}$ Quinn A. Besford, ${ }^{\dagger}$ Yi Ju, ${ }^{\dagger}$ Qi-Zhi Zhong, ${ }^{\dagger}$ Stuart T. Johnston,,${ }^{\S, \perp}$ Jiajing Zhou, ${ }^{\dagger}$ and Frank Caruso ${ }^{*, \dagger}$

${ }^{\dagger}$ ARC Centre of Excellence in Convergent Bio-Nano Science and Technology, and the Department of Chemical Engineering, The University of Melbourne, Parkville, Victoria 3010,

Australia

Bio21 Institute of Molecular Science and Biotechnology, The University of Melbourne, Parkville, Victoria 3010, Australia

${ }^{\S}$ Systems Biology Laboratory, School of Mathematics and Statistics, and the Department of Biomedical Engineering, The University of Melbourne, Parkville, Victoria 3010, Australia ${ }^{\perp}$ ARC Centre of Excellence in Convergent Bio-Nano Science and Technology, Melbourne School of Engineering, The University of Melbourne, Parkville, Victoria 3010, Australia

*E-mail: fcaruso@unimelb.edu.au

KEYWORDS: Plant phenolics, selective assembly, metal-phenolic networks, coordinationdriven assembly, multicomponent systems. 


\begin{abstract}
Selective self-assembly in multicomponent mixtures offers a method for isolating desired components from complex systems for the rapid production of functional materials. Developing approaches capable of selective assembly of "target" components into intended threedimensional structures is challenging because of the intrinsically high complexity of multicomponent systems. Herein, we report the selective coordination-driven self-assembly of metal-phenolic networks (MPNs) from a series of complex multicomponent systems (including crude plant extracts) into thin films via metal chelation with phenolic ligands. The metal (Fe $\left.{ }^{\mathrm{III}}\right)$ selectively assembles low abundant phenolic components (e.g., myricetrin and quercetrin) from plant extracts into thin films. This selective metal-phenolic assembly is independent of the substrate properties (e.g., size, surface charge and shape). Moreover, the high selectivity of our method is consistent across different target phenolic ligands in model mixtures, even though each individual component can form thin films from single component systems. A computational simulation of film formation suggests that the driving force for the selective behavior stems from differences in the number of chelating sites in the phenolic structures. The MPN films are shown to demonstrate improved antioxidant properties compared with the corresponding phenolic compounds in their free form, therefore exhibiting potential as free-standing antioxidant films.
\end{abstract}




\section{Introduction}

Self-assembly in nature affords the formation of complex biological systems from multicomponent mixtures, in which individual components specifically assemble into ordered hierarchical structures. ${ }^{1-4}$ For example, a wide variety of natural protein nanocages (e.g., ferritin, heat shock proteins, virus-like particles) are formed from the selective self-assembly of more than 20 subunits of precursors in complex multicomponent systems in living organisms. ${ }^{5,6}$ These examples in nature have inspired the development of biomimetic approaches using self-assembly as a tool to construct functional synthetic materials for applications including drug delivery, sensing, and catalysis. ${ }^{7-13}$ However, unlike nature, most of these approaches are limited to singlecomponent systems, ${ }^{9,11}$ as control over the self-assembly process in a multicomponent environment has remained challenging owing to the competing interactions of multiple building blocks. ${ }^{14}$

Selective self-assembly from complex multicomponent systems has the potential to provide new ways to engineer materials with unique properties, and has thus drawn widespread interest in recent years. ${ }^{15,16}$ However, the development of selective approaches that can assemble "target" components into defined structures by negating competing interactions with other components within multicomponent systems is challenging. ${ }^{17}$ Several selective assembly techniques have been developed (e.g., orthogonal self-assembly, dynamic imine chemistry). ${ }^{18,19}$ Nitschke and Lehn combined imine chemistry and metal ion coordination to selectively generate metallosupramolecular grid architectures in a mixture of different aminophenol and carbonyl components. ${ }^{20}$ In another study, Yang and co-workers reported the selective assembly of complex tris[2]pseudorotaxanes based on orthogonal metal-ligand coordination and host-guest interactions. $^{21}$ However, these methods generally focused on assembling complexes into 
nanoscale structures. To our best knowledge, approaches for fabricating materials with higher order structures are rare.

Metal-phenolic assembly has been reported as a universal approach for the formation of hybrid films on diverse substrates. ${ }^{22}$ A prominent feature of this assembly approach is its versatility in terms of phenolic ligands that can be used as building blocks, including tannic acid, ${ }^{22}$ flavonoids, ${ }^{23}$ small phenolic ligands, ${ }^{24}$ synthetic polyphenols, ${ }^{25-27}$ and tea infusions. ${ }^{28}$ Herein, the metal-phenolic assembly technique was used to selectively assemble target phenolic compounds into higher order structures (e.g., thin films) from complex multicomponent systems of phenolic species. The results showed that iron ions $\left(\mathrm{Fe}^{\mathrm{III}}\right)$ could produce thin films on particle templates via selective assembly of the target phenolic ligands of low abundance $(<2 \%)$ from complex multicomponent systems (Scheme 1b), although both target and competitor phenolic ligands separately can be assembled into films via $\mathrm{Fe}^{\mathrm{III}}$ coordination. The selectivity was demonstrated using natural eucalyptus (Euc) leaf extracts and a model mixture of phenolic ligands, and was independent of the properties of the substrate examined (size, shape, and surface charge). A computational model suggests that the driving force of this selective behavior stems from the difference in the number of chelating sites between the target and competitor phenolic ligands. Finally, the antioxidant properties of the metal-phenolic films is demonstrated. The selective MPN assembly thus provides a promising technique for isolating desired phenolic components from complex systems to rapidly prepare antioxidant films. 


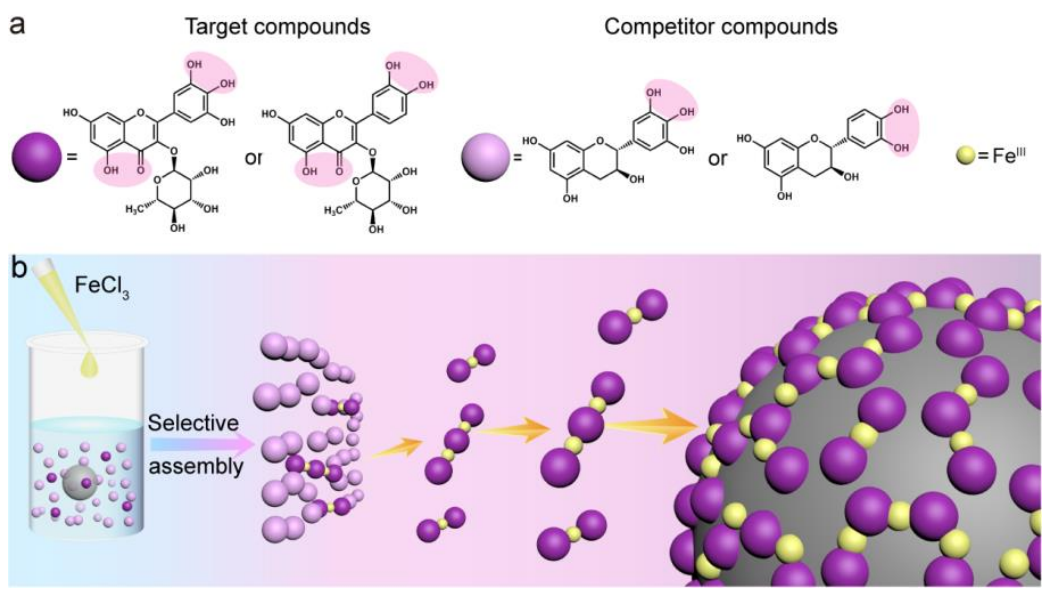

Scheme 1. (a) Molecular structures of target compounds (Myr and Quer) and competitor compounds (Cat and Gal); possible chelating sites for $\mathrm{Fe}^{\mathrm{III}}$ are highlighted in pink. (b) Schematic illustration of selective metal-phenolic assembly on a particle template in a complex multicomponent system.

\section{Experimental Section}

\section{Materials}

Myricetrin (Myr), quercetrin (Quer), and gallocatechin (Gal) were purchased from Carbosynth Pty Ltd (United Kingdom). Catechin (Cat), epigallocatechin gallate (EGCG), iron(III) chloride hexahydrate, poly(diallyldimethylammonium chloride) (PDAC), 2,2'-diphenyl-picrylhydrazyl (DPPH), and $N$-methyl-2-pyrrolidone (NMP) were obtained from Sigma-Aldrich (USA). Poly(methyl methacrylate) (PMMA) particles $(D=105 \pm 4 \mathrm{~nm}$ and $3.69 \pm 0.08 \mu \mathrm{m})$ and aminated silica microparticles $(2.79 \pm 0.12 \mu \mathrm{m})$ were purchased from microParticles $\mathrm{GmbH}$ (Germany). Quartz plates were purchased from ProSciTech (Australia). Sodium hydroxide $(\mathrm{NaOH})$ and acetone were obtained from Chem-Supply (Australia). Mature Euc leaves were collected from an Eucalyptus tree on The University of Melbourne, Parkville campus, Australia.

\section{Preparation of Euc Leaf Extracts}

Mature Euc leaves (35 pieces, weight: 25-30 g) were washed with tap water and then with 
Milli-Q water at least three times to remove dust. The washed leaves were cut into small pieces (approximately $1 \mathrm{~cm} \times 2 \mathrm{~cm}$ ), placed in $400 \mathrm{~mL}$ Milli-Q water, and incubated in an oven at $85^{\circ} \mathrm{C}$ for $12 \mathrm{~h}$. After cooling to room temperature $\left(20-25^{\circ} \mathrm{C}\right)$, the suspension or extract (Euc extract) was filtered through a membrane filter (pore size: $0.8 \mu \mathrm{m}$ ) and stored at $4{ }^{\circ} \mathrm{C}$ until further use.

\section{Metal-Phenolic Assembly onto Various Substrates Using Euc Extracts}

The preparation of capsules from Euc extracts and iron solution was performed using $\sim 3.7 \mu \mathrm{m}$ PMMA colloidal templates following a previously reported method. ${ }^{28}$ Briefly, $500 \mu \mathrm{L}$ Euc extract was pipetted into a $1.5 \mathrm{~mL}$ tube, followed by the addition of $50 \mu \mathrm{L}$ of $10 \% \mathrm{w} / \mathrm{v}$ PMMA particle templates. After vortex mixing for $15 \mathrm{~s}, 50 \mu \mathrm{L} \mathrm{FeCl}_{3} \cdot 6 \mathrm{H}_{2} \mathrm{O}$ solution $(24 \mathrm{mM})$ was added to the tube, and the mixture was vortexed for another $15 \mathrm{~s}$. Subsequently, the $\mathrm{pH}$ of the mixture was adjusted to 5 by the addition of $\mathrm{NaOH}$ solution $(0.5 \mathrm{M})$, followed by vortex mixing for $30 \mathrm{~s}$. Finally, the PMMA templates were removed by washing with $500 \mu \mathrm{L}$ NMP/acetone $(1: 1 \mathrm{v} / \mathrm{v})$ four times. The Euc $/ \mathrm{Fe}^{\mathrm{IIII}}$ capsules were resuspended in $100 \mu \mathrm{L}$ Milli-Q water for further use.

Coating of the $\sim 100 \mathrm{~nm}$ PMMA particles was carried out using the same protocol as that described above but without template removal.

Coating on $\sim 800 \mathrm{~nm}$ mesoporous silica particles (synthesized by the reported method $^{29}$ ) and $\sim 2.8 \mu \mathrm{m}$ aminated silica were also performed as described above but without template removal and using $2 \mathrm{mg}$ (in $50 \mu \mathrm{L}$ water) of silica particles as templates.

Quartz substrates were also examined as planar substrates. Firstly, a quartz plate $(1 \mathrm{~cm} \times 3 \mathrm{~cm})$ was cleaned by Piranha solution $\left(3: 1 \mathrm{H}_{2} \mathrm{SO}_{4} / \mathrm{H}_{2} \mathrm{O}_{2}\right)$ for 20 min. Caution! Piranha solution is highly corrosive. Extreme care should be taken when handling Piranha solution and only small quantities should be prepared. After drying in a stream of nitrogen, the quartz plate was incubated with PDAC/ $\mathrm{NaCl}$ solution $\left(2 \mathrm{mg} \mathrm{mL}^{-1}\right)$ for $10-20 \mathrm{~min}$ at room temperature $\left(20-25^{\circ} \mathrm{C}\right)$ 
to obtain a positively charged substrate, and then rinsed with Milli-Q water. The precoated quartz plate was placed in a $50 \mathrm{~mL}$ tube, into which $10 \mathrm{~mL}$ Euc extract was introduced. After vortexing for $30 \mathrm{~s}, 1 \mathrm{~mL} \mathrm{FeCl} 3 \cdot 6 \mathrm{H}_{2} \mathrm{O}$ solution $(24 \mathrm{mM})$ was added and the tube was vortexed for $30 \mathrm{~s}$. The $\mathrm{pH}$ of the mixture was then adjusted to 5 by adding $\mathrm{NaOH}$ solution $(0.5 \mathrm{M})$, and the tube was vortexed for $30 \mathrm{~s}$. The coating process was repeated three times. Finally, the coated plate was rinsed and stored in Milli-Q water for characterization.

\section{Preparation of Capsules Using Single-Component Systems}

The preparation of capsules using the phenolic ligands Cat or Gal $\left(\mathrm{Cat} / \mathrm{Fe}^{\mathrm{III}}\right.$ or $\left.\mathrm{Gal} / \mathrm{Fe}^{\mathrm{III}}\right)$ was performed as previously reported..$^{24}$ Cat or Gal solution $(500 \mu \mathrm{L}, 15 \mathrm{mM})$ was added to a $1.5 \mathrm{~mL}$ tube containing $50 \mu \mathrm{L}$ of PMMA particles $(\sim 3.7 \mu \mathrm{m}, 10 \% \mathrm{w} / \mathrm{v})$ in water. Then, $200 \mu \mathrm{L}$ $\mathrm{FeCl}_{3} \cdot 6 \mathrm{H}_{2} \mathrm{O}(30 \mathrm{mM})$ was added to the mixture, followed by gentle vortex mixing for $15 \mathrm{~s}$. Subsequently, the $\mathrm{pH}$ of the mixture was adjusted to 5 by the addition of $\mathrm{NaOH}$ solution $(0.5 \mathrm{M})$, followed by vortex mixing for $30 \mathrm{~s}$. Finally, the PMMA templates were removed by washing with $500 \mu \mathrm{L} \mathrm{NMP/acetone} \mathrm{(1:1} \mathrm{v/v)} \mathrm{four} \mathrm{times.} \mathrm{The} \mathrm{capsules} \mathrm{were} \mathrm{resuspended} \mathrm{in} 100 \mu \mathrm{L}$ MilliQ water for further use.

The preparation of $\mathrm{Myr} / \mathrm{Fe}^{\mathrm{III}}$ capsules was performed using the above method with minor modifications. Briefly, $500 \mu \mathrm{L}$ Myr solution $(0.2 \mathrm{mM})$ was added to a $1.5 \mathrm{~mL}$ tube containing 50 $\mu \mathrm{L}$ of PMMA particles $(\sim 3.7 \mu \mathrm{m}, 10 \% \mathrm{w} / \mathrm{v})$ in water. Then, $50 \mu \mathrm{L} \mathrm{FeCl}_{3} \cdot 6 \mathrm{H}_{2} \mathrm{O}(2 \mathrm{mM})$ was added to the mixture, followed by gentle vortex mixing for $15 \mathrm{~s}$. Subsequently, the $\mathrm{pH}$ of mixture pH was adjusted to 5 by the addition of $\mathrm{NaOH}$ solution $(0.1 \mathrm{M})$. The coating cycle was repeated four times before template removal.

\section{Characterization}

Differential interference contrast (DIC) microscopy images of Euc/Fe $\mathrm{F}^{\mathrm{III}}$ capsules were taken on 
an inverted Olympus IX71 microscope. Ultraviolet-visible (UV-vis) absorption spectra were recorded on a SPECORD 250 PLUS UV-vis spectrophotometer. Atomic force microscopy (AFM) measurements were performed on a JPK NanoWizard II BioAFM. Scans were recorded in tapping mode using MikroMasch silicon cantilevers (NSC/CSC). Morphology and element distribution determination were performed by transmission electron microscopy (TEM) and energy-dispersive X-ray spectroscopy (EDX) on a FEI Tecnai TF20 instrument at an operation voltage of $200 \mathrm{kV}$. For the sample preparation for the AFM and TEM/EDX experiments, $5 \mu \mathrm{L}$ capsule suspensions were allowed to air dry on glass slides (pretreated with Piranha solution) and Formvar carbon-coated copper grids, respectively. X-ray photoelectron spectroscopy (XPS) analysis was conducted on a VG ESCALAB220i-XL spectrometer equipped with a hemispherical analyzer. The energy calibration of XPS was referenced to the C 1s peak at 285.0 $\mathrm{eV}$.

\section{Liquid Chromatography-Mass Spectrometry (LC-MS) Studies}

The organic components of the MPN films were analyzed by LC-MS after disassembling the films. The disassembly process was as follows: ${ }^{28} 100 \mu \mathrm{L}$ of concentrated Euc/Fe $\mathrm{F}^{\mathrm{III}}$-coated particle suspensions $\left(3 \times 10^{7}\right.$ particles $\mathrm{mL}^{-1}$, determined by flow cytometry) was mixed with 500 $\mu \mathrm{L} \mathrm{HCl}(6 \mathrm{M})$, vortexed for $1 \mathrm{~min}$, and kept on a rotating mixer for $10 \mathrm{~min}$. After centrifugation of the HCl-treated suspensions, the supernatants were collected and diluted with $1 \mathrm{~mL}$ of Milli-Q water and lyophilized to remove excess $\mathrm{HCl}$. The lyophilized samples were resuspended in 800 $\mu \mathrm{L}$ acidified methanol $(1 \mathrm{mM})$ and filtered using a membrane filter $(0.8 \mu \mathrm{m})$ before LC-MS analyses.

LC-MS analyses were performed on a liquid chromatography system (Agilent 1200 series) with an Agilent 6520 quadrupole time-of-flight mass spectrometer. For each analysis, $5 \mu \mathrm{L}$ of 
sample (i.e., diluted Euc extracts, $\mathrm{Myr}\left(2 \mu \mathrm{g} \mathrm{mL}^{-1}\right.$ in $\left.\mathrm{H}_{2} \mathrm{O}\right)$, Quer $\left(2 \mu \mathrm{g} \mathrm{mL}^{-1}\right.$ in $\left.\mathrm{H}_{2} \mathrm{O}\right)$, or disassembled capsules) was injected into the system, in which each compound was separated on a reverse phase column $(150 \mathrm{~mm}$ (length) $\times 4.6 \mathrm{~nm}$ (internal diameter); $5 \mu \mathrm{m}$ silica particles; Agilent ZORBAX Eclipse XDB C18), with 100\% Milli-Q water as mobile phase A and 70\% acetonitrile in $0.1 \%$ formic acid $(\mathrm{v} / \mathrm{v})$ as mobile phase B. Detailed parameters can be obtained from our previously reported work. ${ }^{28}$ Electrospray mass spectra were recorded in negative ionization mode over a mass range $m / z$ 100-1000.

A base peak chromatogram of $m / z 200-1000$ was employed to identify compounds in the Euc leaf extracts and disassembled capsules, as the $\mathrm{m} / \mathrm{z}$ ratios of many phenolic compounds are in that range. Combined extracted ion chromatograms (EICs) were used to compare the phenolic compounds detected in disassembled capsules with those in the Euc leaf extracts and model phenolic mixtures.

\section{Modeling}

We developed a simulation to mimic the MPN film formation process, according to our published lattice-based random walk model.$^{30}$ Rather than considering a single type of phenolic compound, in the simulation mimicking the experiments in this work, we include both a target phenolic compound (purple) and a competitor compound with a single chelating site (pink). In the model, it is assumed that the target phenolic compound has a similar binding constant to that of the competitor compound. Both the target phenolic compound and the competitor compound move randomly and may occupy a particular site in the lattice. Compounds in the simulation undergo random motion on a hexagonal lattice and can undergo binding events with other compounds upon contact, provided there are free chelating sites on both compounds and $\mathrm{Fe}^{\mathrm{III}}$ is present in between the two compounds. Once a compound in the simulation binds to another one 
(pink) or two (purple) compounds, it is unable to undergo further binding events. We consider an

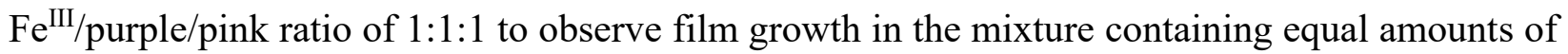
target and competitor compounds. During the assembly process, any compound that has reached the left boundary of the lattice sites is bound to the substrate and can no longer move. $\mathrm{Fe}^{\mathrm{III}}$ ions will move and bind to available chelating sites randomly and participate in further binding events. The thickness of the film as a function of time was monitored during the simulation.

\section{DPPH Assays}

A DPPH assay was performed to evaluate the radical scavenging ability (antioxidant property) of the Euc/Fe ${ }^{\mathrm{III}}$ films. DPPH was freshly prepared before the experiments. Different amounts of Euc/ $\mathrm{Fe}^{\mathrm{III}}$-coated PMMA particles $(\sim 3.7 \mu \mathrm{m})$ as well as a phenolic mixture containing equivalent amounts of Myr and Quer were added to $1 \mathrm{~mL}$ DPPH ethanolic solution $(125 \mu \mathrm{M})$. After incubation for $20 \mathrm{~min}$ and centrifugation ( $2000 \mathrm{~g}, 1 \mathrm{~min}), 200 \mu \mathrm{L}$ supernatant was withdrawn and introduced into a 96-well plate for analysis. The absorbance at $515 \mathrm{~nm}$ was measured. The percentage of remaining DPPH was determined and plotted against the concentration of capsules or Myr/Quer mixture to determine EC50 (concentration of the antioxidant agent required to deplete $50 \%$ of the initial amount of DPPH).

To study the antioxidant properties of the capsules at repeated use or cycles, the final concentration of DPPH was fixed at $60 \mu \mathrm{M}$ by mixing $90 \mu \mathrm{L}$ DPPH ethanolic solution $(700 \mu \mathrm{M})$, $780 \mu \mathrm{L}$ ethanol, and $30 \mu \mathrm{L}$ Euc/Fe $\mathrm{Fe}^{\mathrm{III}}$-coated PMMA particles $(\sim 3.7 \mu \mathrm{m})$. The number of $\mathrm{Euc} / \mathrm{Fe}^{\mathrm{III}}$-coated particles was fixed at $6 \times 10^{6}$ in all DPPH assays. Samples were protected from light and kept on a rotating mixer for $20 \mathrm{~min}$ at $25^{\circ} \mathrm{C}$. Then, $200 \mu \mathrm{L}$ of the particle suspension was placed in a fresh tube and centrifuged $(2000 \mathrm{~g}, 1 \mathrm{~min})$. The supernatant $(150 \mu \mathrm{L})$ was withdrawn and introduced into a $96-$-well plate and the absorbance at $515 \mathrm{~nm}$ was measured. 


\section{Results and Discussion}

Metal-phenolic assembly within a complex multicomponent system was first investigated using natural Euc leaf extracts. Euc extracts were chosen as the multicomponent system in the present study, as they are a rich source of different phenolic compounds including Myr, Quer, Gal, Cat, and chlorogenic acid (CA). ${ }^{31,32}$ The extract was prepared from Euc leaves by a conventional infusion method, ${ }^{22}$ i.e., by incubating cut and washed leaves in Milli-Q water in an oven $\left(85^{\circ} \mathrm{C}\right)$ for $12 \mathrm{~h}$, as shown in Figure $\mathrm{S} 1$. Over time, the original colorless suspension turned slightly yellow, indicating successful extraction of the phenolic compounds from the Euc leaves. LC-MS experiments were performed on the Euc extracts to identify the phenolic compounds present in the system. As shown in Figure 1a, the extract consisted of seven major phenolic compounds CA, Cat, apigenin-7-glucoside (AG), Gal, Myr, Quer, and isorhamnetin-3-Oglucoside (Isg). The chemical structures of these compounds are shown in Figure S2, and Table S1 summarizes their retention times, $\mathrm{m} / \mathrm{z}$, and literature references for their identification. ${ }^{31,33-35}$ Among the compounds identified, Gal was the most abundant ( $50 \%$ area), whereas Myr and Quer were present in relatively smaller amounts in the Euc extract, as shown in Table S1. 

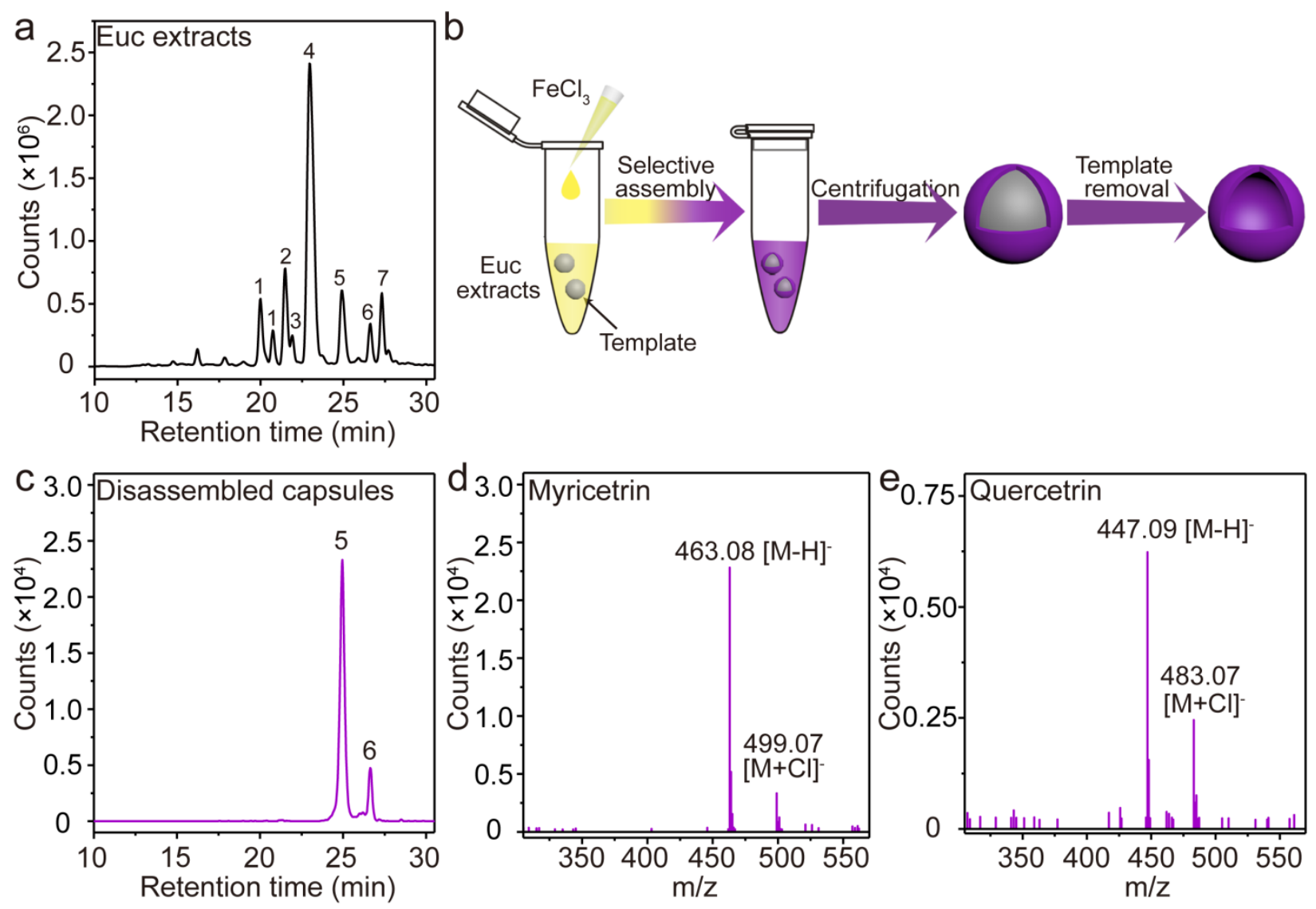

Figure 1. Preparation and characterization of Euc extracts and Euc/Fe ${ }^{\mathrm{III}}$ capsules obtained by selective MPN assembly. (a) Combined extracted ion chromatograms (EICs) of the Euc extract showing retention times of the major compounds: (1) chlorogenic acid (CA); (2) catechin (Cat); (3) apigenin-7-glucoside (AG); (4) gallocatechin (Gal); (5) myricetrin (Myr); (6) quercetrin (Quer); and (7) isorhamnetin 3-O-glucoside (Isg). (b) Schematic illustration of capsule preparation from Euc extracts by selective assembly. (c) Combined EICs of CA ( $m / z$ 353.09), Cat ( $m / z$ 289.07), AG ( $m / z$ 431.19), Gal ( $m / z$ 305.07), Myr ( $m / z$ 463.09), Quer ( $m / z$ 447.09), Isg $(m / z$ 477.23) in the disassembled capsules. Mass spectra of the detected compounds: (d) Myr and (e) Quer.

Using the Euc extract as a mixed phenolic source, MPN film formation was then investigated by a discrete assembly method (i.e., mixing phenolic ligands with an iron salt solution in the presence of template particles). ${ }^{22}$ As shown in Figure 1b, $\mathrm{Fe}^{\mathrm{III}}$ solution was added to the Euc extract solution containing PMMA particles $(D=\sim 3.7 \mu \mathrm{m})$, and the $\mathrm{pH}$ of the mixture was adjusted to 5 (see Experimental Section for details). After mixing and subsequent washing in 
water, MPN film formation was observed by the color darkening with template coating (Figure S3). The templates were removed by using a mixed solution of NMP/acetone $(1: 1 \mathrm{v} / \mathrm{v})$, resulting in stable and monodisperse Euc/Fe ${ }^{\mathrm{III}}$ capsules, as observed in the DIC microscopy image in Figure 2a. The morphology of the monodisperse capsules was further examined by TEM analysis (Figure 2b). AFM analysis revealed monodisperse capsules with folds and creases that are typical features of collapsed capsules (Figure $2 \mathrm{c}$ ). ${ }^{28}$ Figure $2 \mathrm{~d}$ compares the UV-vis spectra of the capsule suspension and the pure extract; a ligand-to-metal charge-transfer (LMCT) band was observed at $565 \mathrm{~nm}$ for the capsules, which corresponds to catechol/Fe $\mathrm{Fe}^{\mathrm{III}}$ coordination. EDX analysis (Figure 2e) confirmed the presence of Fe in the films. The oxidation state of iron in the capsules was further investigated by XPS. As shown in Figure 2f, the core-level spectra of Fe showed $\mathrm{Fe} 2 \mathrm{p}_{3 / 2}$ and $\mathrm{Fe} 2 \mathrm{p}_{1 / 2}$ signals at $\sim 712$ and $\sim 725 \mathrm{eV}$ respectively, suggesting that $\mathrm{Fe}^{\mathrm{III}}$ was the dominant species in the capsules.
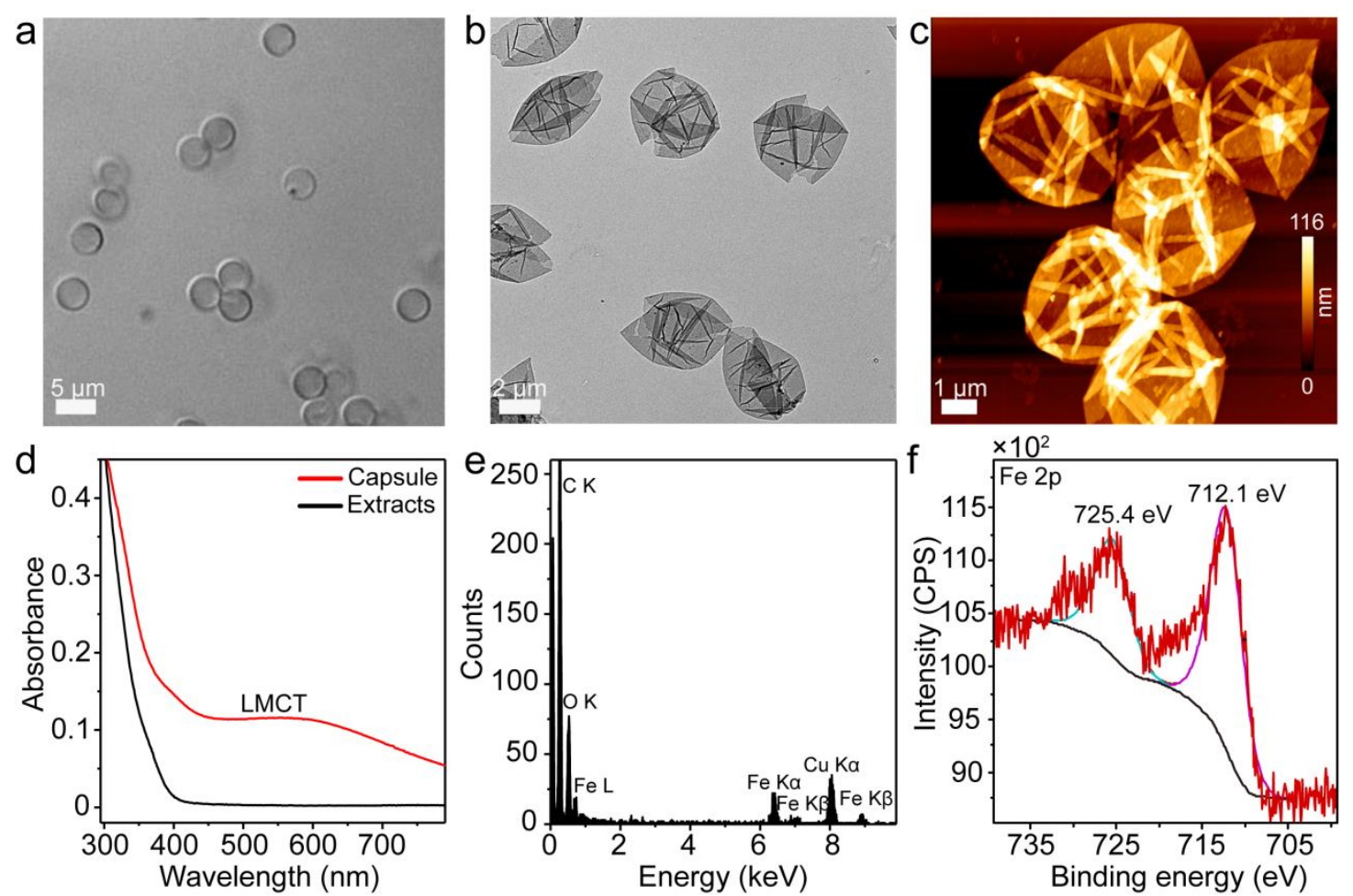

Figure 2. Physicochemical characterization of Euc/Fe $\mathrm{Fe}^{\mathrm{III}}$ capsules prepared by selective metal- 
phenolic assembly from Euc extracts. (a) DIC microscopy, (b) TEM, and (c) AFM images of the Euc/ $\mathrm{Fe}^{\mathrm{III}}$ capsules. (d) $\mathrm{UV}-\mathrm{Vis}$ absorption spectra of the Euc extracts and $\mathrm{Euc} / \mathrm{Fe}^{\mathrm{III}}$ capsule suspension. (e) EDX and (f) XPS spectra of the Euc/Fe $\mathrm{F}^{\mathrm{III}}$ capsules.

To investigate the molecular composition of the Euc $/ \mathrm{Fe}^{\mathrm{III}}$ film, the capsules were disassembled and the components were subjected to LC-MS analyses. Figure 1c shows a combined EICs of the disassembled capsules, where the retention times for the two major peaks were found to be 24.9 and $26.7 \mathrm{~min}$. These peaks were assigned to Myr and Quer respectively by comparing their retention times and MS signals (Figure 1d, e) with their commercial standards (Figure S4). Thus, despite their relatively low abundance, Myr and Quer were found to be the major components of the capsules, suggesting the selective behavior of the metal-phenolic assembly in the Euc leaf extract into thin films. The concentrations of Myr and Quer in the extracts were 67.4 and $20.3 \mu \mathrm{g}$ $\mathrm{mL}^{-1}$, respectively, using the LC-MS standard curves in Figure S5, whereas their concentrations in the capsules were determined to be 0.33 and 0.04 pg per capsule. The Myr-to-Quer mass ratio in the capsules was higher than that in the starting extract (8.2 vs 3.3 ).

The versatility of the present selective assembly method to form MPN films on various substrates with different sizes, structures, surface charge, and shapes (Figure 3a) was also examined. Metal-phenolic film formation was observed on PMMA particles $(\sim 100 \mathrm{~nm})$, mesoporous silica particles ( $\sim 800 \mathrm{~nm}$, negatively charged), aminated silica particles $(\sim 2.8 \mu \mathrm{m}$, positively charged), and planar quartz substrates $(1 \mathrm{~cm} \times 3 \mathrm{~cm})$, as indicated by the color darkening with substrate coating (Figure $3 \mathrm{~b}$ ). When the Euc/Fe $\mathrm{F}^{\mathrm{III}}$ films on these substrates were disassembled and analyzed by LC-MS, two major peaks corresponding to Myr and Quer (Figure S6) were observed in the spectra, indicating that the selective metal-phenolic assembly for film formulation was independent of substrate properties. The Myr-to-Quer ratio in the films 
depended on the substrates (Table S2), possibly because the substrate surface properties impact the adsorption of different MPN complexes.

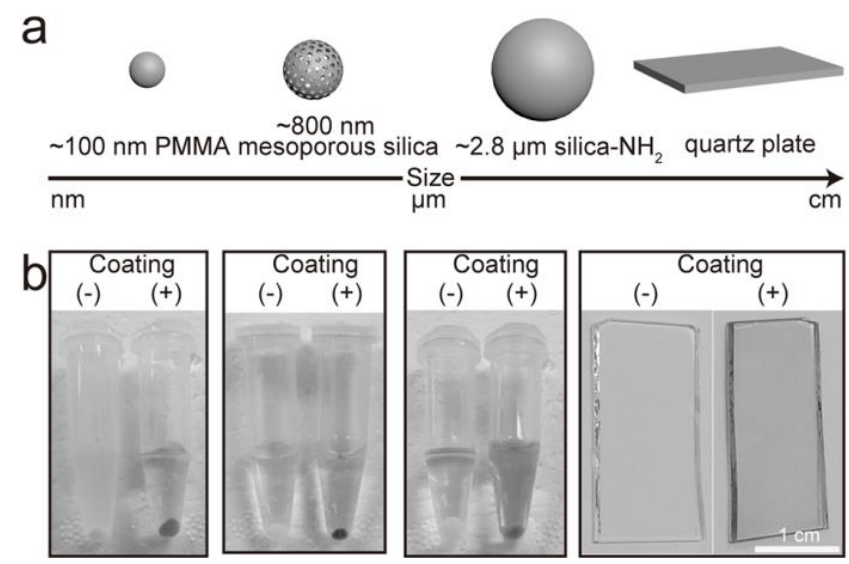

Figure 3. (a) Types of substrates investigated for selective MPN assembly: $\sim 100 \mathrm{~nm}$ PMMA particles, $\sim 800 \mathrm{~nm}$ mesoporous silica particles, $\sim 2.8 \mu \mathrm{m}$ aminated silica particles, and $1 \mathrm{~cm} \times 3$ cm quartz plate. (b) Photographs of the substrates (-) and MPN-coated substrates $(+)$ obtained by selective MPN assembly.

To gain further insight into the present selective metal-phenolic assembly, the assembly experiments were performed using a model phenolic mixture containing Myr, Gal, and Cat (molar ratio of 1:1:1) that mimicked the Euc extract. Myr was the major phenolic component in the assembled films, whereas Gal and Cat were the major components of the starting Euc extract. Note that these three ligands could form MPN films from single-component systems (i.e., where only one type of phenolic ligand is present in the system) as shown in Figure S7, which was in agreement with our previous finding that phenolic compounds possessing at least one catechol group are capable of forming metal-phenolic films. ${ }^{24} \mathrm{Fe}^{\mathrm{III}}$ solution was added to the model multicomponent mixture containing the above three ligands to prepare the MPN films on PMMA particles $(D=\sim 3.7 \mu \mathrm{m})$. The obtained films on PMMA particles were then disassembled and analyzed by LC-MS. Figure 4a shows a combined EICs of the disassembled capsules prepared 
from the model phenolic mixture. The major peak observed at a retention time of $24.9 \mathrm{~min}$ corresponds to Myr (Figure 4b), and no trace signals of Cal and Cat were observed. Thus, Fe ${ }^{\mathrm{III}}$ can selectively assemble Myr in the model mixture into thin films similar to that observed in the natural Euc extract. To confirm the selectivity further, the concentration of the competing phenolic ligands in the mixture was increased $(\mathrm{Myr} / \mathrm{Ca} / \mathrm{Gal}=1: 25: 25)$ such that the Myr molar composition was $<2 \%$. Likewise, the resulting films featured Myr as the dominant phenolic component (Figures S8 and S9), demonstrating selectivity of the assembly even with a low abundant target ligand.
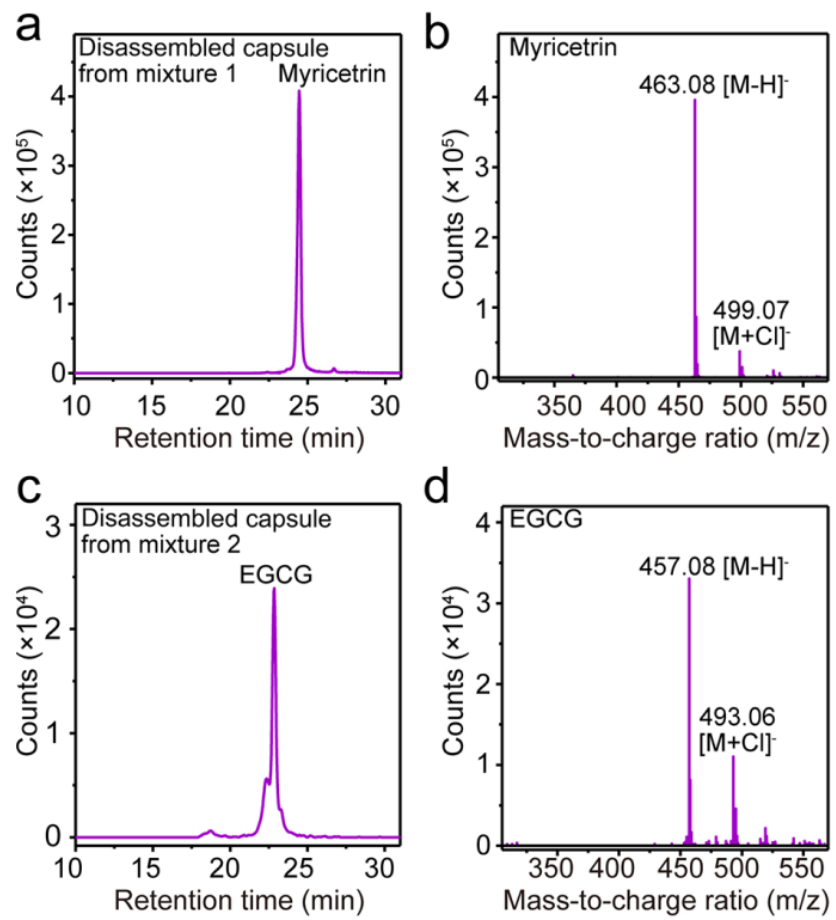

Figure 4. Selective metal-phenolic assembly in model phenolic mixtures. (a) Combined EICs of Cat $(\mathrm{m} / \mathrm{z}$ 289.07), Gal $(\mathrm{m} / \mathrm{z}$ 305.07), Myr ( $\mathrm{m} / \mathrm{z}$ 463.09) in disassembled capsules formed by selective assembly from a mixture containing Myr, Cat, and Gal (Mixture 1). (b) Mass spectra of the detected compound (Myr) in capsules obtained from Mixture 1. (c) Combined EICs of Cat $(\mathrm{m} / z$ 289.07), Gal $(\mathrm{m} / \mathrm{z}$ 305.07), EGCG $(\mathrm{m} / \mathrm{z}$ 457.08) in disassembled capsules formed by selective assembly from a mixture containing, Cat, Gal, and EGCG (Mixture 2). (d) Mass spectra of the detected compound (EGCG) in capsules obtained from Mixture 2. 
Previous studies have shown that the number of chelating sites on ligands can impact on the ability of the ligand to form coordination-driven materials. ${ }^{36,37}$ For example, conventional coordination polymer particles are generally synthesized using bifunctional ligand precursors. ${ }^{36}$ In the present case, the most significant difference between the structure of the target and competitor phenolic compounds was the number of possible $\mathrm{Fe}^{\mathrm{III}}$ chelating sites (shaded in pink in Scheme 1a and Figure S2). From this information, we hypothesize that the number of chelating sites on the phenolic compound is a possible reason for the selective assembly observed in complex multicomponent systems. Both the target and competitor ligands can form metal-phenolic complexes at the early stages of the reaction (Figure 5a). As the reaction proceeds, the target ligands with two chelating sites can grow and form large extendable networks. Owing to the higher number of sites in such networks, surface adsorption and simultaneous cross-linking is more favored than in smaller-sized competitor complexes obtained from compounds with fewer chelating sites. 

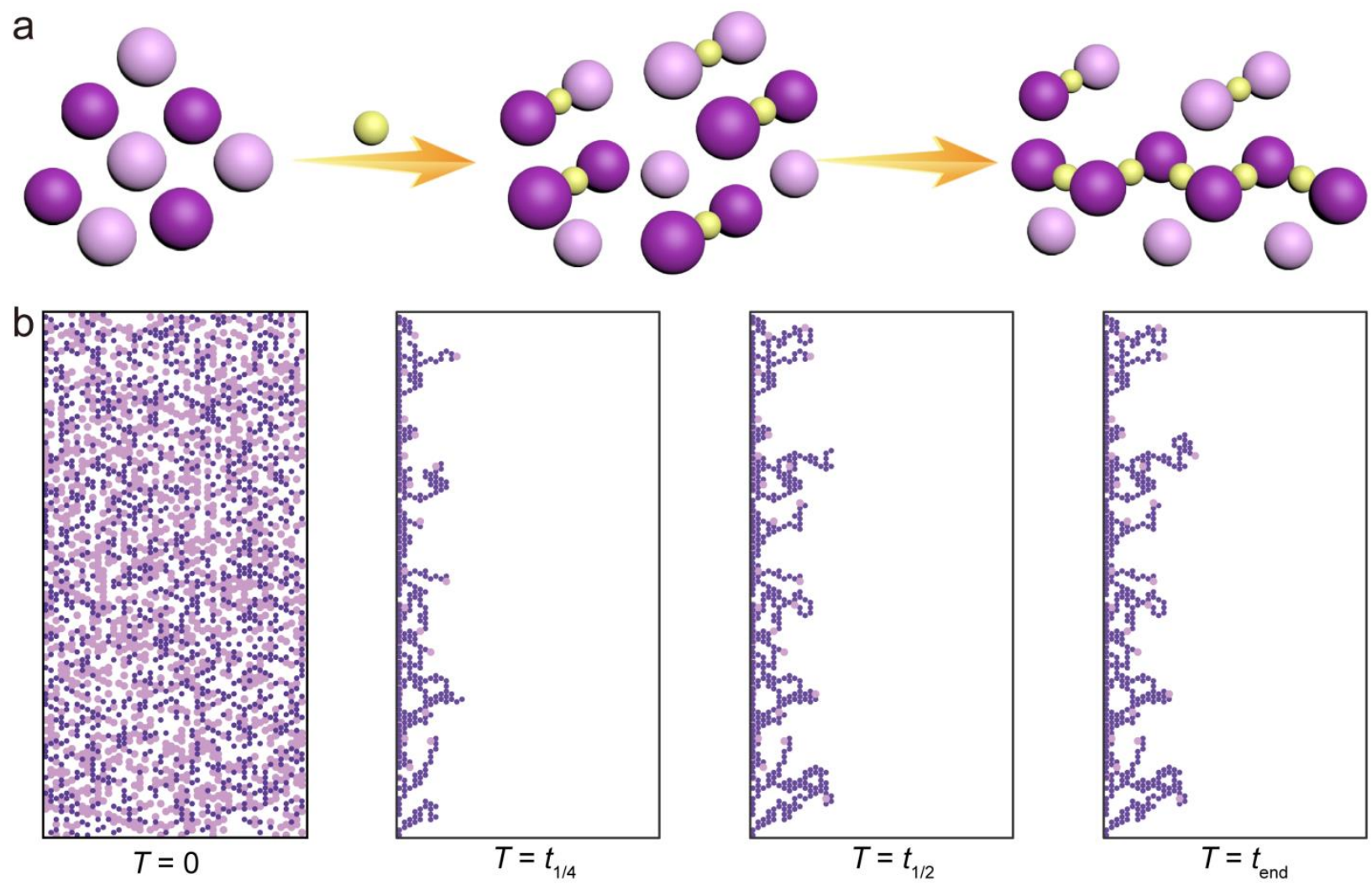

Figure 5. Proposed mechanism and computer modeling of selective MPN assembly. (a) Schematic illustration of the formation of large extendable target compound $/ \mathrm{Fe}^{\mathrm{III}}$ complexes in the presence of small competitor/Fe ${ }^{\mathrm{III}}$ complexes. (b) Computer modeling of selective MPN assembly, in which target compounds with two chelating sites (purple) and competitor compounds with one chelating site (pink) randomly chelate $\mathrm{Fe}^{\mathrm{III}}$ and deposit onto the substrate. At the start of the assembly $(T=0)$, the system contains an equal number of unbound (free) target compound and competitor. At different time points ( $T=t_{1 / 4}, t_{1 / 2}$, and $\left.t_{\text {end }}\right)$, free compounds that are not bound to the left boundary (template) are removed, resulting in the film, which preferably grows with the extendable target compounds that bear a higher number of chelating sites.

To further examine this hypothesis, a computational model simulating the interaction between target phenolic (purple) compounds or competitor compounds with a single chelating site (pink) and $\mathrm{Fe}^{\mathrm{III}}$ ions was constructed. All three species in the model undergo random motion following our recent approach. ${ }^{30}$ The left boundary of the lattice sites represents the template. The target 
phenolic and competitor compounds share a similar binding affinity to $\mathrm{Fe}^{\mathrm{III}}$. The target phenolic compounds can chelate two $\mathrm{Fe}^{\mathrm{III}}$ ions and further bind to other target phenolic compounds or competitors from two sites, provided that a free chelating site remains for both compounds. In comparison, competitors can only chelate one $\mathrm{Fe}^{\mathrm{III}}$ ion, which then binds to target phenolic compounds or other competitors. The model simulation revealed that the film grew with time and that the target phenolic compounds (purple) were the dominant component of the growing film. This selective assembly was time-independent, as observed at points $t_{1 / 4}, t_{1 / 2}$, and $t_{\text {end }}$ (Figure $5 b$ ). This result agrees with our experimental data and the above hypothesis, indicating that despite target and competitor phenolic compounds having similar binding affinities to $\mathrm{Fe}^{\mathrm{III}}$, the difference in the number of $\mathrm{Fe}^{\mathrm{III}}$ chelating sites likely contributes to selective metal-phenolic assembly into thin films. We infer this as the ligands with a higher number of chelation sites are more likely to form the extendable complexes to promote ligand-surface interactions and simultaneous coordination cross-linking for MPN film formation.

To confirm whether this selective assembly is generic or case-specific, the target phenolic ligand was changed from Myr to EGCG (structure shown in Figure S10), as it also possesses two chelation sites but a different structure. Thus, the phenolic mixture contained EGCG, Cat, and Gal at a molar ratio of EGCG/Cat/Gal 1:1:1. When the resulting films were disassembled and analyzed by LC-MS, the major phenolic component in the films was found to be EGCG, as shown in Figure 4c and d. This result also supports the hypothesis that the difference in the number of chelating sites likely determines the selective assembly of the phenolic ligands into thin films from a complex multicomponent system. The selective assembly reported herein can provide several benefits: it offers a route for forming thin films, comprising one or two components with unique properties, from a complex multicomponent system, irrespective of 
substrate properties, and it allows the utilization of low-cost (or freely available) crude natural mixtures to fabricate functional materials with defined composition.

Finally, the antioxidant properties of the selectively assembled films (from Euc extract) were examined on the basis of their radical scavenging ability. DPPH assays were used, which measure the reduction in the absorbance of the radical DPPH at $515 \mathrm{~nm}$ or a color change from purple to yellow in the presence of an antioxidant compound. ${ }^{23,38}$ When the Euc/Fe $\mathrm{F}^{\mathrm{III}}$-coated PMMA particles were added to ethanolic DPPH solution, a color change from purple to yellow was observed (Figure S11), indicating the reduction of the DPPH radicals (Figure S12). Figure 6a shows that the characteristic absorption peak of DPPH at $515 \mathrm{~nm}$ significantly decreased upon addition of the film to the ethanolic DPPH solution, indicating that the film can scavenge the free radicals. EC50 (i.e., antioxidant concentration at which 50\% radical DPPH scavenging occurs) was measured for the films as well as for an equivalent mixture of free Myr and Quer (Myr/Quer at mass ratio of $8.3: 1$ ) by plotting radical scavenging activity against concentration (Figure S13). ${ }^{39}$ As observed in Figure 6b, the equivalent mixture of free Myr and Quer displayed an EC50 of $17.5 \mu \mathrm{M}$, whereas the capsules featured a lower EC50 of $16.4 \mu \mathrm{M}$, indicating the slightly improved antioxidant behavior obtained in the presence of capsules when compared with the corresponding free phenolic compounds. The improvement could possibly be due to the iron center that can stabilize the semiquinone radical intermediate (Figure S14) ${ }^{23,40}$ In addition, the Euc/Fe $\mathrm{F}^{\mathrm{III}}$-coated PMMA particles could be used in consecutive cycles as investigated by subjecting the particles to three DPPH scavenging cycles. The first cycle resulted in almost complete DPPH depletion. After washing the particles in ethanol, the Euc/Fe $e^{\mathrm{III}}-$ coated PMMA particles could still scavenge $\sim 70 \% \mathrm{DPPH}$, and after the third cycle, $50 \% \mathrm{DPPH}$ radical depletion was observed (Figure 6c). In summary, the selectively assembled MPN films displayed 
enhanced and antioxidant capacity in consecutive cycles, and thus may be applied as particle- or film-based radical scavenging agents.
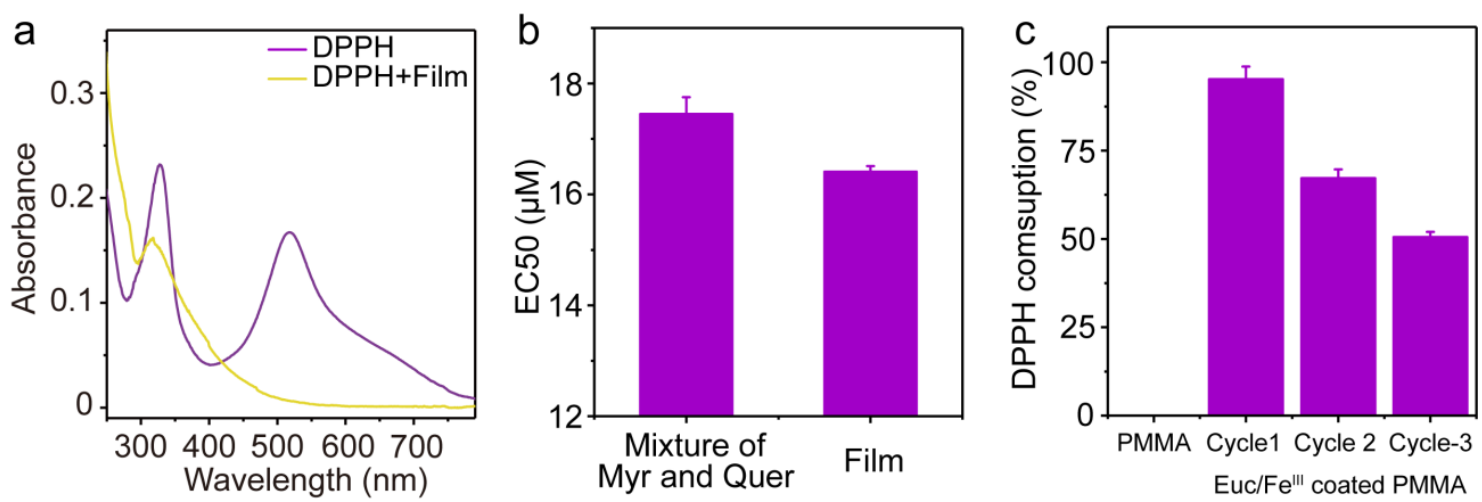

Figure 6. Radical scavenging ability of Euc/Fe $\mathrm{E}^{\mathrm{III}}$ films. (a) UV-Vis spectra of DPPH (30 $\mu \mathrm{M}$ in ethanol) and the supernatant collected from DPPH treated with films. (b) EC50 of Euc/Fe $\mathrm{Fe}^{\mathrm{III}}$ coated PMMA particles and equivalent mixture of Myr and Quer. (c) Repeated radical scavenging activity of the Euc/Fe $\mathrm{F}^{\mathrm{III}}$-coated PMMA particles over three cycles of incubation (20 min) with intermediate washing steps; bare PMMA particles were also tested as a control.

\section{Conclusion}

We have shown the selective self-assembly between $\mathrm{Fe}^{\mathrm{III}}$ and specific ligands on various substrates from complex plant polyphenol mixtures. The present selective metal-phenolic assembly can recognize small variations in the number of possible chelating sites between different phenolic compounds. The assembly is robust and versatile — the same phenomenon was observed in model mixtures of commercial phenolic compounds and crude natural extracts such as Euc leaf extracts and the assembly could be performed on various substrates with different size, shape, and surface properties. The high selectivity of this approach and its applicability to various phenolic mixtures make it a potentially powerful tool for pharmaceutical separation, whereby specific compounds need to be isolated from complex mixtures. Finally, the films obtained from selective metal-phenolic assembly exhibit improved radical scavenging properties 
in consecutive cycles (when compared with free phenolic compounds), rendering them a potential candidate for antioxidant application.

\section{ASSOCIATED CONTENT}

Supporting Information. LC-MS analyses, DIC microscopy images of capsules, chemical structures of major phenolic compounds in Euc extract, DPPH scavenging assays.

\section{AUTHOR INFORMATION}

\section{Corresponding author}

*E-mail: fcaruso@unimelb.edu.au

\section{Acknowledgements}

This research was conducted and funded by the Australian Research Council Centre (ARC) of Excellence in Convergent Bio-Nano Science and Technology (project number CE140100036) and an ARC Discovery Project (DP170103331). F.C. acknowledges the award a National Health and Medical Research Council Senior Principal Research Fellowship (APP1135806). This work was performed in part at the Materials Characterisation and Fabrication Platform (MCFP) at The University of Melbourne and the Victorian Node of the Australian National Fabrication Facility (ANFF). We also acknowledge Sergey Rubanov from the Bio21 Advanced Microscopy Facility (The University of Melbourne) for assistance with TEM.

\section{References}

1. Whitesides, G. M.; Grzybowski, B. Self-Assembly at All Scales. Science 2002, 295, $2418-2421$.

2. Mattia, E.; Otto, S. Supramolecular Systems Chemistry. Nat. Nanotechnol. 2015, 10, $111-119$.

3. $\quad$ Ariga, K.; Nishikawa, M.; Mori, T.; Takeya, J.; Shrestha, L. K.; Hill, J. P. Self-Assembly as a Key Player for Materials Nanoarchitectonics. Sci. Technol. Adv. Mater. 2019, 20, 51-95.

4. Hiraoka, S. Unresolved Issues that Remain in Molecular Self-Assembly. Bull. Chem. Soc. 
Jpn. 2018, 91, 957-978.

5. Lee, E. J.; Lee, N. K.; Kim, I.-S. Bioengineered Protein-Based Nanocage for Drug Delivery. Adv. Drug Delivery Rev. 2016, 106, 157-171.

6. Murata, S.; Yashiroda, H.; Tanaka, K. Molecular Mechanisms of Proteasome Assembly. Nat. Rev. Mol. Cell Biol. 2009, 10, 104-115.

7. Pattni, B. S.; Chupin, V. V.; Torchilin, V. P. New Developments in Liposomal Drug Delivery. Chem. Rev. 2015, 115, 10938-10966.

8. Cabral, H.; Miyata, K.; Osada, K.; Kataoka, K. Block Copolymer Micelles in Nanomedicine Applications. Chem. Rev. 2018, 118, 6844-6892.

9. Yan, X.; Zhu, P.; Li, J. Self-Assembly and Application of Diphenylalanine-Based Nanostructures. Chem. Soc. Rev. 2010, 39, 1877-1890.

10. Zhu, L.; Liu, X.-Q.; Jiang, H.-L.; Sun, L.-B. Metal-Organic Frameworks for Heterogeneous Basic Catalysis. Chem. Rev. 2017, 117, 8129-8176.

11. Bucknall, D. G.; Anderson, H. L. Polymers Get Organized. Science 2003, 302, 19041905.

12. Furukawa, H.; Cordova, K. E.; O’Keeffe, M.; Yaghi, O. M. The Chemistry and Applications of Metal-Organic Frameworks. Science 2013, 341, 1230444.

13. Liu, S.; Tang, Z. Nanoparticle Assemblies for Biological and Chemical Sensing. J. Mater. Chem. 2010, 20, 24-35.

14. Pappas, C. G.; Shafi, R.; Sasselli, I. R.; Siccardi, H.; Wang, T.; Narang, V.; Abzalimov, R.; Wijerathne, N.; Ulijn, R. V. Dynamic Peptide Libraries for the Discovery of Supramolecular Nanomaterials. Nat. Nanotechnol. 2016, 11, 960-967.

15. Okesola, B. O.; Mata, A. Multicomponent Self-Assembly as a Tool to Harness New Properties from Peptides and Proteins in Material Design. Chem. Soc. Rev. 2018, 47, 3721-3736.

16. Liu, Y.; Liu, B.; Nie, Z. Concurrent Self-Assembly of Amphiphiles into Nanoarchitectures with Increasing Complexity. Nano Today 2015, 10, 278-300.

17. Jacobs, W. M.; Frenkel, D. Self-Assembly of Structures with Addressable Complexity. $J$. Am. Chem. Soc. 2016, 138, 2457-2467.

18. Hu, X.-Y.; Xiao, T.; Lin, C.; Huang, F.; Wang, L. Dynamic Supramolecular Complexes Constructed by Orthogonal Self-Assembly. Acc. Chem. Res. 2014, 47, 2041-2051.

19. Belowich, M. E.; Stoddart, J. F. Dynamic Imine Chemistry. Chem. Soc. Rev. 2012, 41, 2003-2024.

20. Nitschke, J. R.; Lehn, J.-M. Self-Organization by Selection: Generation of a Metallosupramolecular Grid Architecture by Selection of Components in a Dynamic Library of Ligands. Proc. Natl. Acad. Sci. U. S. A. 2003, 100, 11970-11974.

21. Wang, W.; Zhang, Y.; Sun, B.; Chen, L.-J.; Xu, X.-D.; Wang, M.; Li, X.; Yu, Y.; Jiang, W.; Yang, H.-B. The Construction of Complex Multicomponent Supramolecular Systems via the Combination of Orthogonal Self-Assembly and the Self-Sorting Approach. Chem. Sci. 2014, 5 , 4554-4560.

22. Ejima, H.; Richardson, J. J.; Liang, K.; Best, J. P.; van Koeverden, M. P.; Such, G. K.; 
Cui, J.; Caruso, F. One-Step Assembly of Coordination Complexes for Versatile Film and Particle Engineering. Science 2013, 341, 154-157.

23. Bertleff-Zieschang, N.; Rahim, M. A.; Ju, Y.; Braunger, J. A.; Suma, T.; Dai, Y.; Pan, S.; Cavalieri, F.; Caruso, F. Biofunctional Metal-Phenolic Films from Dietary Flavonoids. Chem. Commun. 2017, 53, 1068-1071.

24. Rahim, M. A.; Kempe, K.; Müllner, M.; Ejima, H.; Ju, Y.; van Koeverden, M. P.; Suma, T.; Braunger, J. A.; Leeming, M. G.; Abrahams, B. F.; Caruso, F. Surface-Confined Amorphous Films from Metal-Coordinated Simple Phenolic Ligands. Chem. Mater. 2015, 27, 5825-5832.

25. Ju, Y.; Cui, J.; Müllner, M.; Suma, T.; Hu, M.; Caruso, F. Engineering Low-Fouling and pH-Degradable Capsules through the Assembly of Metal-Phenolic Networks. Biomacromolecules 2015, 16, 807-814.

26. Ju, Y.; Cui, J.; Sun, H.; Müllner, M.; Dai, Y.; Guo, J.; Bertleff-Zieschang, N.; Suma, T.; Richardson, J. J.; Caruso, F. Engineered Metal-Phenolic Capsules Show Tunable Targeted Delivery to Cancer Cells. Biomacromolecules 2016, 17, 2268-2276.

27. Besford, Q. A.; Ju, Y.; Wang, T.-Y.; Yun, G.; Cherepanov, P.; Hagemeyer, C. E.; Cavalieri, F.; Caruso, F. Self-Assembled Metal-Phenolic Networks on Emulsions as Low-Fouling and pHResponsive Particles. Small 2018, 14, 1802342.

28. Rahim, M. A.; Björnmalm, M.; Bertleff-Zieschang, N.; Ju, Y.; Mettu, S.; Leeming, M. G.; Caruso, F. Multiligand Metal-Phenolic Assembly from Green Tea Infusions. ACS Appl. Mater. Interfaces 2018, 10, 7632-7639.

29. Wang, J.-G.; Zhou, H.-J.; Sun, P.-C.; Ding, D.-T.; Chen, T.-H. Hollow Carved SingleCrystal Mesoporous Silica Templated by Mesomorphous Polyelectrolyte-Surfactant Complexes. Chem. Mater. 2010, 22, 3829-3831.

30. Yun, G.; Besford, Q. A.; Johnston, S. T.; Richardson, J. J.; Pan, S.; Biviano, M.; Caruso, F. Self-Assembly of Nano- to Macroscopic Metal-Phenolic Materials. Chem. Mater. 2018, 30, 5750-5758.

31. Santos, S. A. O.; Freire, C. S. R.; Domingues, M. R. M.; Silvestre, A. J. D.; Neto, C. P. Characterization of Phenolic Components in Polar Extracts of Eucalyptus globulus Labill. Bark by High-Performance Liquid Chromatography-Mass Spectrometry. J. Agric. Food Chem. 2011, 59, 9386-9393.

32. Mota, I.; Rodrigues Pinto, P. C.; Novo, C.; Sousa, G.; Guerreiro, O.; Guerra, Â. R.; Duarte, M. F.; Rodrigues, A. E. Extraction of Polyphenolic Compounds from Eucalyptus globulus Bark: Process Optimization and Screening for Biological Activity. Ind. Eng. Chem. Res. 2012, 51, 6991-7000.

33. Hossain, M. B.; Rai, D. K.; Brunton, N. P.; Martin-Diana, A. B.; Barry-Ryan, C. Characterization of Phenolic Composition in Lamiaceae Spices by LC-ESI-MS/MS. J. Agric. Food Chem. 2010, 58, 10576-10581.

34. de Brito, E. S.; Pessanha de Araújo, M. C.; Lin, L.-Z.; Harnly, J. Determination of the Flavonoid Components of Cashew Apple (Anacardium occidentale) by LC-DAD-ESI/MS. Food Chem. 2007, 105, 1112-1118. 
35. Chen, Y.; Yu, H.; Wu, H.; Pan, Y.; Wang, K.; Jin, Y.; Zhang, C. Characterization and Quantification by LC-MS/MS of the Chemical Components of the Heating Products of the Flavonoids Extract in Pollen Typhae for Transformation Rule Exploration. Molecules 2015, 20, $18352-18366$.

36. Spokoyny, A. M.; Kim, D.; Sumrein, A.; Mirkin, C. A. Infinite Coordination Polymer Nano- and Microparticle Structures. Chem. Soc. Rev. 2009, 38, 1218-1227.

37. Novio, F.; Simmchen, J.; Vázquez-Mera, N.; Amorín-Ferré, L.; Ruiz-Molina, D. Coordination Polymer Nanoparticles in Medicine. Coord. Chem. Rev. 2013, 257, 2839-2847.

38. Deligiannakis, Y.; Sotiriou, G. A.; Pratsinis, S. E. Antioxidant and Antiradical $\mathrm{SiO}_{2}$ Nanoparticles Covalently Functionalized with Gallic Acid. ACS Appl. Mater. Interfaces 2012, 4, 6609-6617.

39. Cherrak, S. A.; Mokhtari-Soulimane, N.; Berroukeche, F.; Bensenane, B.; Cherbonnel, A.; Merzouk, H.; Elhabiri, M. In Vitro Antioxidant versus Metal Ion Chelating Properties of Flavonoids: A Structure-Activity Investigation. PLoS One 2016, 11, e0165575.

40. Moridani, M. Y.; Pourahmad, J.; Bui, H.; Siraki, A.; O’Brien, P. J. Dietary Flavonoid Iron Complexes as Cytoprotective Superoxide Radical Scavengers. Free Radical Biol. Med. 2003, 34, 243-253. 
Table of Contents Use Only

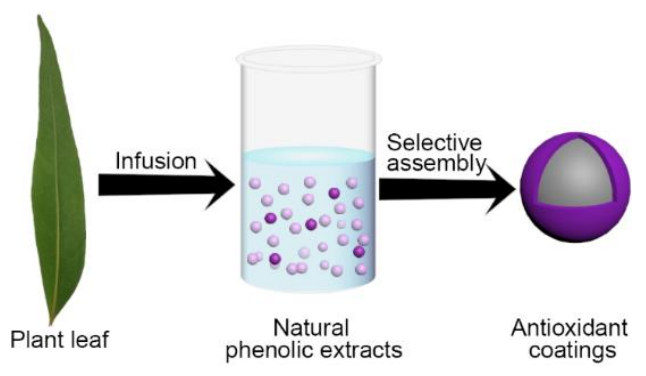

